\title{
Multipolling and OFDMA Reservation Protocol for IEEE 802.11 Networks
}

\author{
David Pérez ${ }^{1}$, José Luis Valenzuela ${ }^{2}$, Javier Villares ${ }^{3}$ \\ Universitat Politècnica de Catalunya (UPC) \\ Avda. Canal Olímpic s/n, (08860) Castelldefels, SPAIN \\ ${ }^{1}$ dperez@tsc.upc.edu, ${ }^{2}$ valensetsc.upc.edu, ${ }^{3}$ javi@t.sc.upc.edu
}

\begin{abstract}
This paper presents an efficient multipolling scheme that improves the IEEE 802.11 MAC protocol efficiency and does not suffer from the hidden terminal problem or inefficient channel usage problems. To design an efficient polling scheme, the access point (AP) needs to obtain information about the current transmission status and channel state for each station. In this new scheme, we propose the use of multipolling frames in the downlink and Orthogonal Frequency Division Multiple Access (OFDMA) for the uplink reservation frames in order to reduce signaling overhead.
\end{abstract}

\section{INTRODUCTION}

Wireless networks have experienced a vertiginous evolution and are used all around the world. Especially IEEE 802.11 based wireless networks. Since its appearance several amendments have been done to the standard, whose objective is generally to increase the transmission rates. However, in order to be able to support voice and multimedia applications and high density of users, just increasing transmission rates is not enough. It is also necessary a good management of MAC resources and to provide users with quality of service (QoS).

To guarantee QoS to 802.11 users, the 802.11e amendment [1] defines a new access mechanism that has two (possibly concurrent) modes of operation: enhanced distributed channel access (EDCA), which is a contention-based channel access function and, Hybrid Coordination Function controlled channel access (HCCA), which is a polling mechanism usually coordinated by the access point. As it is well-known, the main drawback of EDCA is that it cannot provide QoS in realtime communications because of the traffic-dependent latency of the adopted contention-based medium access mechanism. Moreover, this problem is specially critical when the network becomes congested because EDCA does not support any mechanism of admission control. To tackle this deficiency and provide multimedia communications with QoS, HCCA replaces the EDCA contention-based access procedure by a centralized reservation-based medium access strategy. However, a major issue of HCCA is that it is inefficient when only a small number of stations have packets to transmit due to the inefficient adopted polling method and the large signaling overhead it requires.

Several MultiPolling (MP) schemes have been proposed in [2] or [3] to reduce the overhead of polling frames. Nevertheless, in those contributions, if a polled station does not have enough pending frames to transmit during the assigned time duration, the remaining time is wasted. To solve this problem, [4] proposes a two-step multipolling (TS-MP) MAC protocol including a status collection period in which those stations having traffic to transmit report this status to the AP. TS-MP employs Time Division Multiple Access to implement the channel reservation requiring an excessive overhead during the collection period as the number of active users increases. This drawback motivated this new parallel reservation strategy based on OFDMA that increases the efficiency of TS-MP and makes it practically independent of the number of active users. Also, like other well-known protocols (EDCA, HCCA), the proposed protocol is easily adaptable to the $802.11 \mathrm{~g}$ physical layer (PHY), what makes it compatible with $802.11 \mathrm{~g}$ stations.

\section{Proposed Multipolling Protocol}

In this section a novel multipolling reservation mechanism with OFDMA station response is presented (MPR-OFDMA). This solution takes advantage of the high efficiency of the multipoll mechanisms as well as introduces priority demand for each station. In the HCCA, the AP sends an individual polling frame to every STA in the polling list. Clearly, the number of polling frames can be reduced if a multipolling mechanism is used. In this paper, we propose an efficient multipolling mechanism that has the advantages of efficient channel utilization and low implementation overhead.

With this proposal the AP can obtain information from each station in every Contention Free Period (CFP). Using this information, the AP efficiently schedules the polling sequence and assigns the Transmission Opportunities (TXOP) to stations. Fig. 1 shows the basic scheme of the proposed protocol which divides the CFP into five intervals:

1.- At the beginning of the CFP, the AP transmits a Multipoll request (MPR) frame, to poll some of the stations registered in the polling list based on a given scheduling policy.

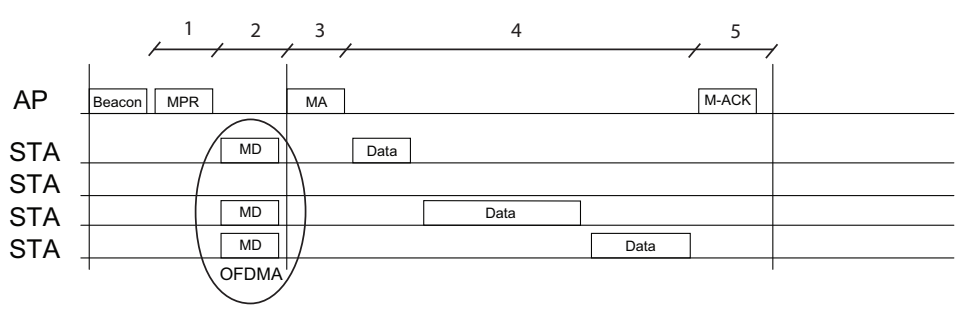

Fig. 1. Multipoll packet exchange 


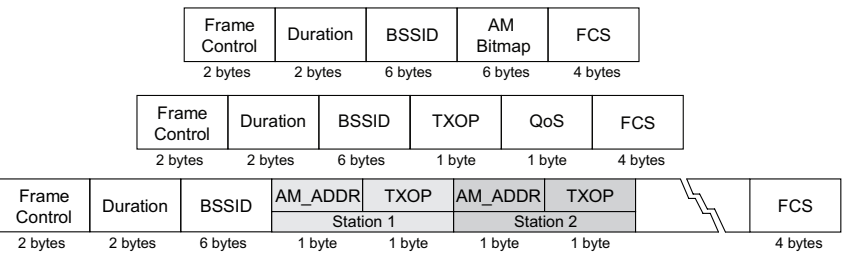

Fig. 2. Multipoll Request, Multipoll Demand and Multipoll Assign Frames

2.- Each station polled by the AP sends a Multipoll Demand frame to the AP with some status information using and OFDMA technique.

3.- After receiving the frame, the AP sends a Multipoll Assignation frame that schedules the terminals transmissions.

4.- In this step the stations proceed to do their transmissions.

5.- Finally the transmissions of each station are acknowledged all together by means of a single frame.

Each step is analyzed with detail in the following sections.

\section{A. Multipoll Request}

First, the AP transmits a single frame at the bitrate used by the slowest polled station ensuring that all the associated stations can decode correctly the MPR frame. Its structure is depicted in Fig. 2. All the fields of the frame follow the 802.11 standard, except for the new Active Member (AM) Bitmap field.

During the association process the AP numbers each associated station. Aftar that the AP decides which stations to poll following a convenient scheduling algorithm. In a first approach, a maximum of 48 associated stations is considered corresponding to the data subcarriers of the 802.11a/g OFDM physical layer. Each bit of the AM Bitmap field represents an associated station. So six bytes are needed to map 48 stations. The aim of this field is that stations can know the exact number of stations of the QBSS that are being polled.

\section{B. Multipoll Demand}

At a second phase the polled stations have to indicate their QoS requirements. Using the information of the AM bitmap field, the 48 OFDM carriers are distributed among the stations. Then each station uses only a subset of the 48 subcarriers to transmit the Multipoll Demand frame. For example, with just one active station, this STA could use the 48 carriers to transmit its frame. With two, 24 carriers are available per station and so on. The assignment of subcarriers is ordered following the numbering adopted during the association process.

In this way all the stations transmit their needs simultaneously and we avoid to wait for a station that does not respond or does not have anything to transmit. All the stations transmit at the rate of the previous multipoll request frame because the station with the worst link defines the MD frame duration.

The frame format is shown in Fig. 2. In this frame the new fields are: Transmission Opportunity (TXOP) and QoS. Each field has a length of one byte. The TXOP and QoS fields indicate the time and priority needs of each station, respectively.

\section{Multipoll Assignation}

After analyzing and managing the needs of the different stations, the AP responds with a variable length frame indicating the granted radio resources. This frame should also be transmitted at the rate of the least favored user.

Fig. 2 shows the structure of this frame. After the standard Frame control, Duration, and BSSID fields, the AP introduces two fields per station. The first field Active Member Address (AM_ADDR) identifies the targeted station. And the TXOP field denotes the transmission time given to that station. These two fields are repeated for each station that answered the polling. The frame ends with a Frame Check Sequence.

\section{Data Transmission}

During the data transmission phase each station transmits orderly as scheduled in the Multipoll Assignation phase. A SIFS time must be left between transmissions. The stations are allowed to transmit at different rates. However, they must not exceed their assigned TXOP. No acknowledgment frames must be transmitted during this phase.

\section{E. Acknowledgement}

Finally, a multiple acknowledgement frame is transmitted. This frame is similar to the MPR shown in Fig. 2. In the AM Bitmap field a positive bit indicates the successful reception of all the frames transmitted in the TXOP assigned to a given station.

\section{THE OFDM ACCESS MECHANISM}

The proposed OFDMA multipoll reservation mechanism can be deployed after simple upgrades in the $802.11 \mathrm{~g}$ standardized physical layer (PHY). First of all, terminals should be able to connect and disconnect those subcarriers indicated by the AM Bitmap field in the MPR frame. Also, the pseudorandom sequence transmitted in the pilot subcarriers should be configurable to facilitate the AP to estimate the channel frequency response and carrier frequency offset associated to every active terminal. Finally, although the interleaver and channel encoder could be optimized for the proposed OFDMA air interface, it seems a priori feasible to apply the existing convolutional coding and interleaving to the group of subcarriers associated to individual users.

Beyond these straightforward modifications, the principal obstacle to adapt the 802.11g PHY layer for the uplink multipoll scheme proposed in this article is the severe synchronization requirements imposed by the OFDMA access mechanism. Ideally, all the terminals should adjust their transmission time and frequency to guarantee the orthogonality of subcarriers is preserved at the receiver side, i.e., at the access point (AP). To do so, the uplink transmissions are synchronized by means of the beacon frame broadcasted by the AP at the beginning of the CFP. Unfortunately, this conventional "open-loop" synchronization cannot compensate for the propagation delay and Doppler frequency offset associated to the polled stations. To deal with these two synchronization errors, 


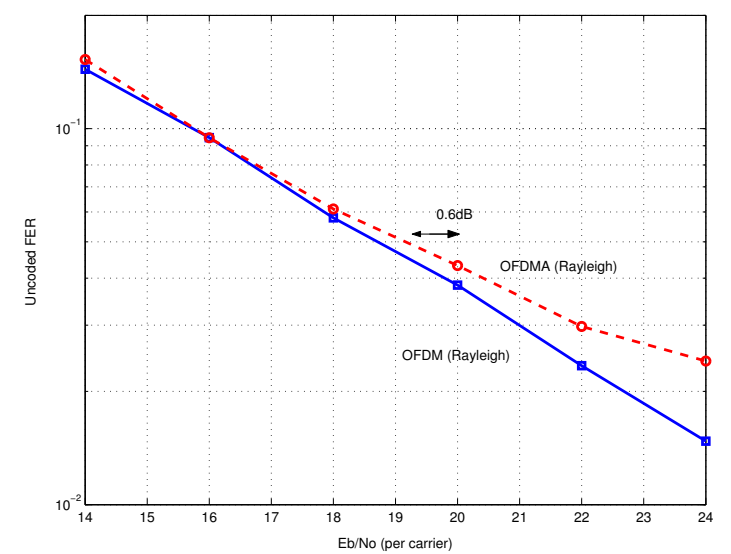

Fig. 3. Simulation of the uncoded/raw FER of the $802.11 \mathrm{~g}$ physical layer in a Rayleigh fading channel (6Mbps, BPSK). The lower curve (square markers) corresponds to a synchronous scenario whereas the upper curve (circle markers) corresponds to the asynchronous OFDMA scenario under study. In both cases, an exponential power delay profile is simulated with delay spread of $100 \mathrm{~ns}$ and maximum round-trip delay equal to $500 \mathrm{~ns}$

it would be necessary to implement an additional "closedloop" synchronization mechanism allowing the AP to adjust remotely the transmission time and frequency of every polled station. However, after some preliminary simulations of the $802.11 \mathrm{~g}$ physical layer, we conclude that "closed-loop" time and frequency synchronization can be avoided even in rather stringent propagation scenarios.

To validate the reduced impact of these synchronization errors on the performance of the OFDMA access, two pessimistic outdoor scenarios have been evaluated in terms of the uncoded frame error rate (FER). In these simulations, we have considered that 48 stations are answering the multipoll request frame. The transmission rate of all the stations is the one of the slowest one (worst channel state) and, implicitly, we are considering that all the stations perform power control to reduce intercarrier interference (ICI) and power consumption. In addition, the AP is supposed to carry out estimation and tracking of all the active users channels. To achieve this, the preamble of the multipoll reservation frame is processed to obtain initial channel estimates that are tracked throughout the frame duration using those pilot subcarriers close to the frequency band assigned to every user or, alternatively, by means of blind estimation techniques [5].

In the first scenario, the robustness of the 802.11g PHY in front of time synchronization errors is studied (Fig.3). A worst-case scenario is simulated in which the user of interest is near the AP and the remaining 47 interfering users are located 75 meters away from the AP corresponding to a maximum relative round-trip delay of $500 \mathrm{~ns}$. We assume perfect power control and the lowest transmission rate for all the stations $(6$ Mbps, BPSK). Also, we consider independent none-line-ofsight Rayleigh channels with exponential power delay profile and delay spread equal to $100 \mathrm{~ns}$. This channel model has been selected because it is representative of a typical open area as, for example, an outdoor car-parking [6]. This scenario has

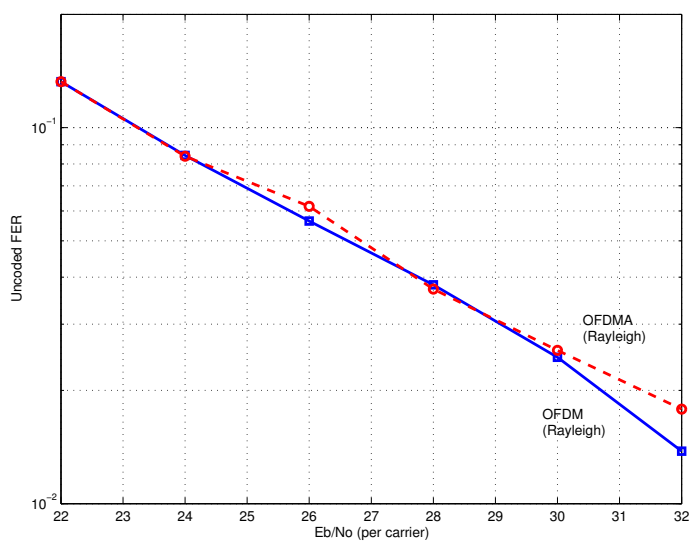

Fig. 4. Simulation of the uncoded/raw FER of the $802.11 \mathrm{~g}$ physical layer in a Rayleigh fading channel (54Mbps, 64-QAM). The lower curve (square markers) corresponds to a synchronous scenario whereas the upper curve (round markers) corresponds to the asynchronous OFDMA scenario under study. In both cases, an exponential power delay profil is simulated with delay spread of $100 \mathrm{~ns}$ and maximum round-trip delay equal to 50ns.

been simulated concluding that the synchronization losses do not exceed $0.6 \mathrm{~dB}$ for any practical $\mathrm{Eb} /$ No lower than $20 \mathrm{~dB}$ (Fig.3).

In the second scenario, the impact of frequency errors on the 802.11g PHY performance is analyzed (Fig.4). In this case, we consider that the 48 users are near the AP (less than 7 meters) and the transmission rate is maximum (54Mbps, 64-QAM). The reasoning is that high-dimensional constellations are more sensitive to synchronization errors than low-dimensional ones. Again, we consider the same channel model but now terminals are moving at 20 kilometers per hour with a maximum Doppler frequency of $44 \mathrm{~Hz}(2.4 \mathrm{GHz}$ band). A pessimistic scenario is studied in which the users Doppler deviation is chosen in order to maximize the intercarrier interference (ICI). Furthermore, we have also taken into account that the uplink oscillators of the polled stations are not perfectly synchronized as studied in [7]. In this scenario, simulations conclude that synchronization losses are negligible for realistic working Eb/No provided that the channel amplitude and phase tracking is correctly performed at the receiver side (Fig. 4).

\section{Throughput CAlculation}

For the EDCA case, the results are obtained using the ns-2 network simulator. For the rest of the solutions the throughput is calculated analytically as explained next.

\section{A. $H C C A$}

The effective packet transmission time is calculated following the scheme of Fig. 5 and then:

$$
\begin{aligned}
T_{e f}(\mu s)= & (P I F S-S I F S) \\
& +N_{o k} \cdot\left(S I F S+T_{p k t}(P O L L)+S I F S+T_{p k t}(D A T A)\right) \\
& +N_{k o} \cdot\left(P I F S+T_{p k t}(P O L L)\right) \\
& +S I F S+T_{p k t}(C F e n d)
\end{aligned}
$$




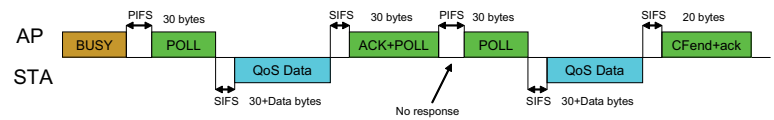

Fig. 5. HCCA packet exchange

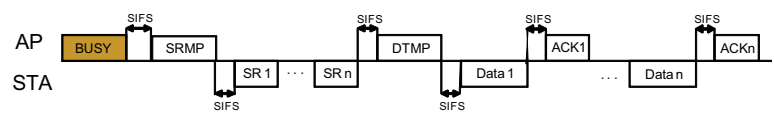

Fig. 6. TS-MP packet exchange

where,

$N_{o k}=$ number of users answering the poll

$N_{k o}=$ number of users not responding

$P O L L=$ bytes of a poll frame $(30)$

$C F e n d=$ bytes of a CFend + ACK frame $(20)$

and,

$$
\begin{aligned}
T_{p k t}(x \text { bytes }) & =P L C P_{\text {preamble }}+\text { SIGNAL+SIGNAL extension } \\
& +\left\lceil\frac{8 \cdot x+\text { Service }+ \text { Tail }}{N_{D B P S}}\right\rceil \cdot T_{\text {sym }}
\end{aligned}
$$

In (2), $N_{D B P S}$ is the number of bits per OFDM symbol and depends on the transmission rate. The operator ceiling $(\lceil\rceil)$ returns the smallest integer greater than or equal the specified number.

The throughput is then calculated using

$$
T h_{H C C A}=N_{o k} \cdot \frac{8 \cdot D A T A}{T_{e f}} \cdot 10^{6}
$$

\section{B. $T S-M P$}

The analysis for TS-MP is similar to the previous one but follows the scheme depicted in Fig. 6

Following the naming used in [4], the effective time is then,

$$
\begin{aligned}
T_{e f}(\mu s)= & S I F S+T_{p k t}(S R M P) \\
& +N_{\text {users }} \cdot\left(\operatorname{SIFS}+T_{p k t}(S R)\right) \\
& \left.+ \text { SIFS }+T_{p k t}(D T M P)\right) \\
& +N_{\text {ok }} \cdot\left(\operatorname{SIFS}+T_{p k t}(D A T A)+\operatorname{SIFS}+T_{p k t}(A C K)\right)
\end{aligned}
$$

where,

$N_{\text {users }}=$ total number of users

$N_{o k}=$ number of users answering the poll

$S R M P=$ bytes of the SRMP frame (37)

$S R=$ bytes of a SR frame (17)

$D T M P=$ bytes of the DTMP frame (73)

$A C K=$ bytes of an ACK frame (14)

$T_{p k t}(x)$ is defined in (2)

And so, the throughput is:

$$
T h_{T S-M P}=N_{o k} \cdot \frac{8 \cdot D A T A}{T_{e f}} \cdot 10^{6}
$$

\section{MPR-OFDMA}

Finally, for our solution the calculations are based on the scheme of Fig. 1 and its effective time is:

$$
\begin{aligned}
T_{e f}(\mu s)= & P I F S+T_{p k t}(M P R) \\
& +S I F S+T_{M D} \\
& \left.+S I F S+T_{p k t}(M A)\right) \\
& +N_{o k} \cdot\left(S I F S+T_{p k t}(D A T A)\right) \\
& +S I F S+T_{p k t}(M-A C K)
\end{aligned}
$$

where,

$$
\begin{aligned}
& N_{\text {users }}=\text { total number of users } \\
& N_{o k}=\text { number of users answering the poll } \\
& M P R=\text { bytes of the MPR frame }(20) \\
& M A=\text { bytes of the MA frame }\left(14+2 \cdot N_{\text {users }}\right) \\
& M-A C K=\text { bytes of the M-ACK frame }(20) \\
& T_{p k t}(x) \text { is defined in }(2)
\end{aligned}
$$

On the other hand,

$$
\begin{aligned}
T_{M D}= & P L C P_{\text {preamble }}+S I G N A L+S I G N A L_{\text {extension }} \\
& +\left\lceil\frac{48}{\left\lfloor 48 / N_{\text {users }}\right\rfloor}\right\rceil \cdot\left\lceil\frac{8 \cdot M D+\text { Service }+ \text { Tail }}{N_{D B P S}}\right\rceil \cdot T_{\text {sym }}
\end{aligned}
$$

The factor $\left[\frac{48}{\left[48 / N_{\text {users }}\right\rfloor}\right]$ denotes the increase of duration due to the increment of users. If there is only one user it will employ the 48 carriers to transmit the frame. Two users will employ 24 carriers each so the duration will be the double and so on.

And finally the throughput is:

$$
T h_{M P R-O F D M A}=N_{o k} \cdot \frac{8 \cdot D A T A}{T_{e f}} \cdot 10^{6}
$$

\section{Results}

In this section the throughput obtained with HCCA, TS-MP and MPR-OFDMA is compared. Figure 7 shows the results obtained for HCCA, the TS-MP and our multipoll solution.

Packets are generated in a Constant Bit Rate (CBR) basis and all the polled stations have always at least one packet to be transmitted. 48 transmitting stations at $54 \mathrm{Mbps}$ rates are considered. The represented throughput is the sum of the contribution of all stations and the horizontal axis indicates the data length of the packets sent.

It can be observed that all HCCA, TS-MP and MPROFDMA respond much better than EDCA. It should be remarked that, in the EDCA case, 48 stations compete for the channel so the number of collisions is high. Thus, EDCA achieves nearly half the throughput obtained by the other access techniques.

Although TS-MP achieves better results than EDCA, the overhead introduced in the polling procedure when 48 users are transmitting is excessive, and even the standard HCCA performs better in that case. It has to be remarked that, for 


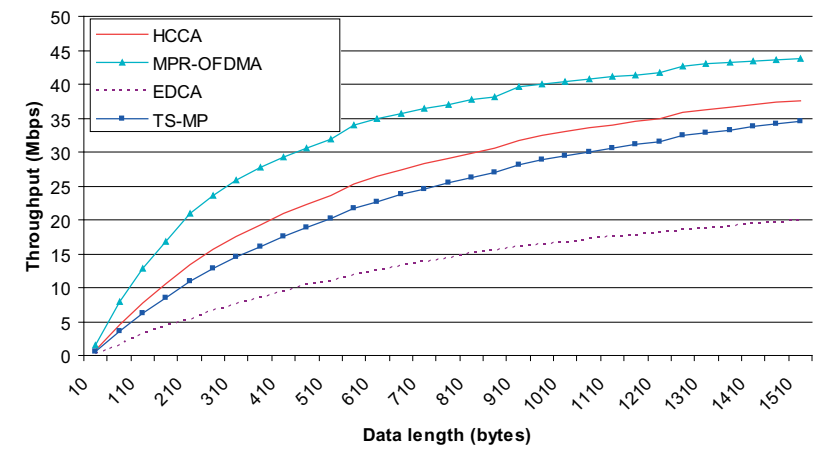

Fig. 7. Compared throughput at $54 \mathrm{Mbps}$

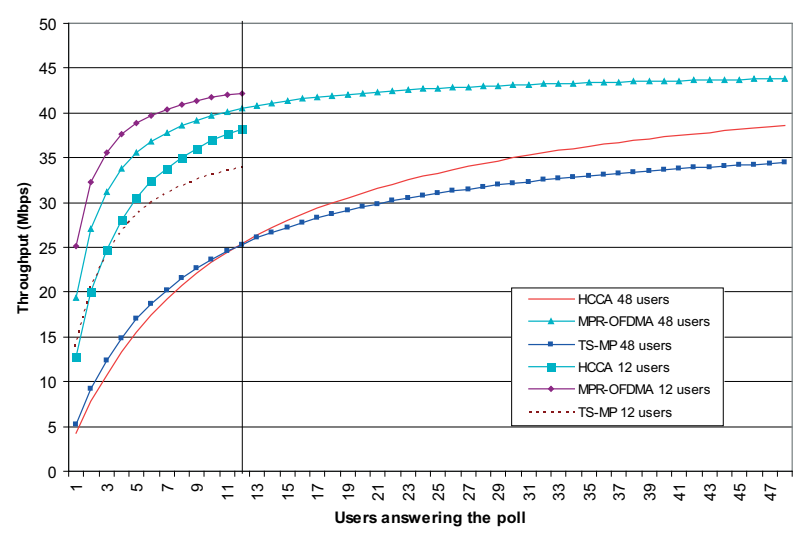

Fig. 8. Throughput when some stations have nothing to transmit at $54 \mathrm{Mbps}$

the HCCA throughput calculations, polling frames include the ACK to the previous received frame to reduce overhead.

Comparing the throughput of HCCA and MPR-OFDMA at $54 \mathrm{Mbps}$, MPR-OFDMA yields a $14 \%$ increase for long packets. This improvement is quasi-constant for data lengths over 250 bytes. For shorter data lengths, time intended for control and management is excessive compared with the employed for data transmissions and, consequently, throughput goes to zero rapidly.

At lower data rates this improvement is not as noticeable. All the solutions except EDCA obtain more or less the same performance. However the MPR-OFDMA is always slightly superior, especially for data lengths between one hundred and six hundred bytes.

Figure 8 depicts the throughput achieved when some of the polled 48 stations do not have frames to transmit. The data length considered is 1500 bytes. It can be observed that the TS-MP performs better than HCCA when less than 13 users answer positively to the poll. However, when the number of transmitting stations increases, the overhead introduced reduces its performance. On the other hand, MPR-OFDMA outperforms both HCCA and TS-MP no matter how many stations answer the poll. However the highest efficiency occurs when just a few stations apply in the reservation period.

Inside figure 8 it is also represented the performance when only 12 stations are polled. As less signalling is needed in all the cases the throughput achieved is higher. The behavior of the 3 scenarios when not all the stations answer to the poll is similar to the explained previously. However, we can see that although MPR-OFDMA obtains always better performance than HCCA or TS-MP, the improvement is more noticeable for higher loads.

Using lower transmission rates the enhancements are not as noticeable, but they still exist. At lower transmission rates, the duration of the frames is higher, so the quantity of data introduced in the time we won by using MPR-OFDMA is lower.

\section{CONCLUSIONS}

In this paper a novel proposal to enhance the 802.11e MAC is proposed. It is based on a multipolling mechanism with an OFDMA uplink. All the stations declare their intentions simultaneously during the multipoll demand phase.

The performance is compared with other multipolling solutions and also with the standard HCCA/EDCA 802.11e modes. The results show that this solution performs better than the rest in all the studied scenarios with different system loads and packet sizes, percentage of users answering to the poll, etc.

The OFDMA nature of this proposal avoids the time spent waiting for polled stations that does not have anything to transmit as happens in HCCA and introduces less overhead than other solutions like TS-MP.

\section{ACKNOWLEDGMENT}

This work was supported in part by the Spanish Ministry of Education and Science and FEDER funds (TEC2007-68094C02-02 and TEC2006-09109) and by the Catalan Government under the grant (2005SGR-00639).

\section{REFERENCES}

[1] IEEE802.11-WG, “Ieee std 802.11e-2005: "ieee standard for information technology-telecommunications and information exchange between systems-local and metropolitan area networks-specific requirements part 11: Wireless lan medium access control (mac) and physical layer (phy) specifications: Amendment 8: Medium access control (mac) quality of service enhancements",' 2005.

[2] S. Lo, G. Lee, and W. Chen, "An efficient multipolling mechanism for ieee 802.11 wireless lans," IEEE TRANSACTIONS ON COMPUTERS, pp. 764-778, 2003.

[3] O. Sharon and E. Altman, "An efficient polling mac for wireless lans," IEEE/ACM Transactions on Networking (TON), vol. 9, no. 4, pp. 439451, 2001.

[4] B. Kim, S. Kim, Y. Fang, and T. Wong, "Two-step multipolling mac protocol for wireless lans," IEEE JOURNAL ON SELECTED AREAS IN COMMUNICATIONS, vol. 23, no. 6, 2005.

[5] S. Wu, S. Lee, and K. Lai, "Joint channel tracking and map detection for ofdma systems in fading channels," Acoustics, Speech and Signal Processing, 2007. ICASSP 2007. IEEE International Conference on, vol. 3, 2007.

[6] A. Kemp and E. Bryant, "Channel sounding of industrial sites in the 2.4 ghz ism band," Wireless Personal Communications, vol. 31, no. 3, pp. 235-248, 2004.

[7] S. Chang, E. Powers, M. Inc, and T. Austin, "Efficient frequency-offset estimation in ofdm-based wlan systems," Electronics Letters, vol. 39, no. 21 , pp. $1554-1555,2003$. 